\title{
HIGH PRESSURE - HIGH TEMPERATURE DIFFRACTION STUDY OF MnTe USING SYNCHROTRON RADIATION
}

\author{
W. Paszkowicz, E. Dynowska \\ Institute of Physics, Polish Academy of Sciences \\ Al. Lotników 32/46, 02-668 Warsaw, Poland \\ AND T. PEUN
}

GeoForschungsZentrum Potsdam, Telegrafenberg A17, 14473 Potsdam, Germany

\begin{abstract}
High pressure - high temperature experiments for MnTe of NiAs type were performed using a synchrotron radiation as X-ray source and a cubic anvil X-ray diffraction press to produce nonambient sample environment. In the investigated range of pressure (0-67.5 kbar) on uploading and with following temperature rise from 296 to $1273 \mathrm{~K}$ (at $67.5 \mathrm{kbar}$ ), the structure type of $\mathrm{MnTe}$ is conserved. An earlier reported nonlinear behaviour of lattice-parameter $c_{0}$ is not found in the present study. Possible reasons of this discrepancy are discussed. The value of bulk modulus and its pressure derivative were calculated from the pressure-volume dependence by fitting the Birch-Murnaghan equation.
\end{abstract}

PACS numbers: $62.50 .+\mathrm{p}, 64.30 .+\mathrm{t}, 65.70 .+\mathrm{y}$

\section{Introduction}

$\mathrm{MnTe}$ is a component of semiconductor solid solutions known as diluted magnetic semiconductors which are extensively studied in many laboratories (for a review see [1]). These compounds crystallize mainly in sphalerite and wurtzite type tetrahedral structures. Phase diagram investigations [2-4] have shown the presence of polymorphism of MnTe. MnTe-I (or $\alpha$-MnTe), stable at ambient temperature and pressure, crystallizes in NiAs-type hexagonal structure. A magnetic transition at $310 \mathrm{~K}$ is known for this form [5]. Apart of a commonly known high-temperature form, $\beta$ - $\mathrm{MnTe}$ of $\mathrm{NaCl}$ type, two other high-temperature forms, $\gamma$ and $\delta$, of unknown structure types have been indicated by differential thermal analysis (DTA) [3]. The interest in studies of $\mathrm{MnTe}$ is due to the predicted and experimentally confirmed possibility of growing the metastable sphalerite-type $\mathrm{MnTe}$ by molecular beam epitaxy (see [6] and references therein). 
Previous experimental high-pressure investigations include studies of compressibility [7-9] and phase transformations [9]. The variation of lattice constants reported for MnTe-I in the stability range of $0-130 \mathrm{kbar}$ in Ref. [7] is characterized by a large scatter of experimental points. In a study in the range of $0-70 \mathrm{kbar}$ [8], a nonlinear behaviour of lattice parameter $c_{0}$ has been found with a minimum at $30 \mathrm{kbar}$ and a maximum at $42 \mathrm{kbar}$; anomalies in temperature dependence of $a_{0}$ and $c_{0}$ at pressures of 28 and $43 \mathrm{kbar}$ have also been reported there. The observed anomalies have been concluded in the cited reference to be possibly due to a magnetic transition. In Ref. [9], two structural phase transitions at $65 \pm 35 \mathrm{kbar}$ to MnTe-II of an unknown structure (probably related to NiAs type) and at $210 \pm 30 \mathrm{kbar}$ to MnTe-III of MnP type structure have been found. The analysis of the above cited results led to a conclusion that to better understand the high-pressure and high-temperature structural changes in MnTe-I, a study using a synchrotron radiation source would be useful. Such a preliminary study of lattice-parameters behaviour within the limits imposed by the experimental setup is presented below, with the aim of determining the compressibility and thermal expansion in the available range of temperatures and pressures.

\section{Experimental}

In the present work, the high pressure - high temperature experiments were performed using synchrotron radiation as X-ray source. The experimental setup consisted in a cubic anvil X-ray diffraction press, MAX80 (described, e.g., in Ref. [10]) and a germanium solid state detector of resolution $155 \mathrm{eV}$ at $5.9 \mathrm{keV}$ installed at F2 beam line at DESY-HASYLAB. The MAX80 facility had similar properties to that used in the earlier high-pressure studies of MnTe [8], but the experiment differed in $\mathrm{X}$-ray source, sample mounting and data collection system.

The sample was prepared from a MnTe-I crystal grown by crystallization from the vapour phase. The crystal was finely grounded and pressed, the investigated specimen volume was approximately $0.5 \mathrm{~mm}^{3}$. The specimen was built from thin horizontal wafers containing single phases of $\mathrm{MnTe}$ and $\mathrm{NaCl}$ marker separated by a $\mathrm{BN}$ layer. The wafers were mounted in a $\mathrm{BN}$ cylinder inside a vertical cylindrical graphite heater located in the middle of a cube made of boron-epoxy mixture. The wafer of $\mathrm{NaCl}$ was used to determine the pressure according to the $\mathrm{NaCl}$ high-pressure equation of state [11], the estimated accuracy was $\pm 1 \mathrm{kbar}$. The measurement of temperature (with accuracy $\pm 10 \mathrm{~K}$ ) was performed with the help of a NiCrSi/NiSi thermocouple mounted within the cube.

The characteristic feature of applied experimental setup is using high-energy white radiation combined with a low fixed diffraction angle. The diffraction angle $\theta$ fixed at $2.9979^{\circ}$ caused that the effective range of studied interplanar spacings extended down to $1.6 \AA$. The peak positions were determined by a profile fitting program assuming a Gaussian shape. The lattice parameters were calculated by Cohen's least squares method (with updated version of the program described in Refs. $[12,13])$ using the $002,101,102,110,103,112,201,004$ and 202 reflections a vailable in the studied energy range; in some cases the 002 peak had to be omitted because of too low intensity. 


\section{Results and discussion}

A preliminary laboratory experiment using a conventional Bragg-Brentano diffractometer showed that there is no trace of secondary phases in the $\mathrm{MnTe}$ crystal. The powder patterns obtained at non-ambient conditions are shown in Fig. 1. The presented patterns are similar in form and quality to the only published high-pressure pattern of $\mathrm{MnTe}$-I [9] measured in the angle-resolved mode at $73 \mathrm{kbar}$; the difference in relative peak intensities between the patterns of Fig. 1 and Ref. [9] is attributed to the effect of preferred orientation of the sample.

In the pressure range 0-67.5 kbar on uploading (at ambient temperature) and with the following pressure rise from 296 to $1273 \mathrm{~K}$ (at $67.5 \mathrm{kbar}$ ), the structure type of NiAs type is found to be conserved. Additional peaks characteristic of MnTe-II appear neither during the uploading (in agreement with the results of Ref. [9]) nor during the following pressure rise. Weak peaks were concluded, on the basis of comparison with the laboratory powder-diffraction pattern, to be the
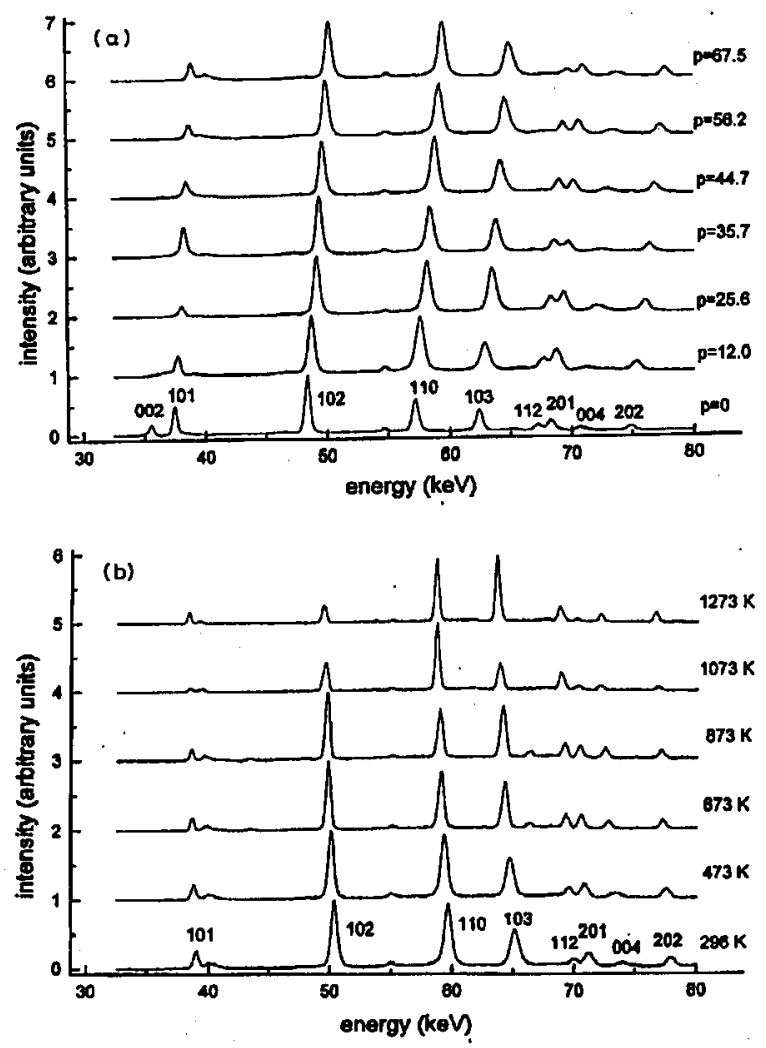

Fig. 1. Powder patterns of MnTe at non-ambient conditions in the pressure range of $0-67.5 \mathrm{kbar}$ on uploading at $296 \mathrm{~K}$ (a) and in the temperature range of $296-1273 \mathrm{~K}$ at 67.5 kbar (b). 
escape peak (that at $39-40 \mathrm{keV}$ ) or to be due to sample mounting or fluorescence (that at about $55 \mathrm{keV}$ ); small features at about 43 and $66.5 \mathrm{keV}$ appearing only at 673 and $873 \mathrm{~K}$ are not identified. With increasing pressure and temperature, a peak-broadening effect is observed. The peak-shape behaviour shows that when the high pressure is fixed at $67.5 \mathrm{kbar}$, the lattice relaxation occurs upon heating, resulting in a visible reduction of the peak width.

\section{TABLE}

Unit-cell dependence of pressure and temperature for MnTe-I during uploading at $296 \mathrm{~K}$ (the first run) and following temperature rise at $67.5 \mathrm{kbar}$ (the second run).

\begin{tabular}{c|c|c|c|l}
\hline \hline $\begin{array}{c}p \\
{[\mathrm{kbar}]}\end{array}$ & $\begin{array}{c}T \\
{[\mathrm{~K}]}\end{array}$ & $\begin{array}{c}a_{0} \\
{[\AA]}\end{array}$ & $\begin{array}{c}c_{0} \\
{[\AA]}\end{array}$ & $\begin{array}{c}V \\
{\left[\AA^{3}\right]}\end{array}$ \\
\hline 0 & 297 & $4.147(2)$ & $6.711(3)$ & $99.92(14)$ \\
12.0 & 296.5 & $4.121(1)$ & $6.652(3)$ & $97.84(9)$ \\
25.6 & 296 & $4.087(2)$ & $6.570(4)$ & $95.04(15)$ \\
35.7 & 296 & $4.064(2)$ & $6.541(4)$ & $93.56(15)$ \\
44.7 & 296 & $4.038(3)$ & $6.502(5)$ & $91.83(21)$ \\
56.2 & 296 & $4.015(4)$ & $6.458(8)$ & $90.17(29)$ \\
67.5 & 296 & $3.994(3)$ & $6.425(6)$ & $88.75(22)$ \\
\hline 67.5 & 296 & $3.982(2)$ & $6.432(8)$ & $88.32(20)$ \\
67.5 & 473 & $4.005(3)$ & $6.465(6)$ & $89.81(22)$ \\
67.5 & 673 & $4.017(1)$ & $6.518(2)$ & $91.09(7)$ \\
67.5 & 873 & $4.022(1)$ & $6.543(1)$ & $91.66(6)$ \\
67.5 & 1073 & $4.035(2)$ & $6.578(4)$ & $92.75(15)$ \\
67.5 & 1273 & $4.045(3)$ & $6.584(6)$ & $93.29(22)$
\end{tabular}

The compression behaviour of MnTe (cf. Table) allowed us to obtain the bulk modulus of the NiAs-type phase. The bulk modulus and its first derivative calculated by fitting the Birch-Murnaghan equation [14] are $B_{0}=473 \mathrm{kbar}$ and $\mathrm{d} B_{0} / \mathrm{d} p=3.0$, respectively. The result of the fitting is presented in Fig. 2. The bulk modulus obtained in this work for MnTe-I is slightly lower than that measured jointly for MnTe-I and MnTe-II (497 kbar) and that of MnTe-III (584 kbar), both reported in Ref. [9]. This sequence of values supports the expectation that high-pressure phases are stiffer than low-pressure phases. The value of bulk modulus for MnTe-I may be compared with that of sphalerite-type MnTe films: it is considerably higher than the value for a film, $B_{0}=151 \mathrm{kbar}$, reported in Ref. [15] as well as that which could be derived by extrapolation of (Cd,Mn)Te thin films data [16], $B_{0}=390 \mathrm{kbar}$ (the derived $\mathrm{d} B_{0} / \mathrm{d} p$ being equal 3.4).

The dependences of unit-cell constants on temperature at fixed $67.5 \mathrm{kbar}$ pressure are quoted in Table. They complete earlier investigations performed at 


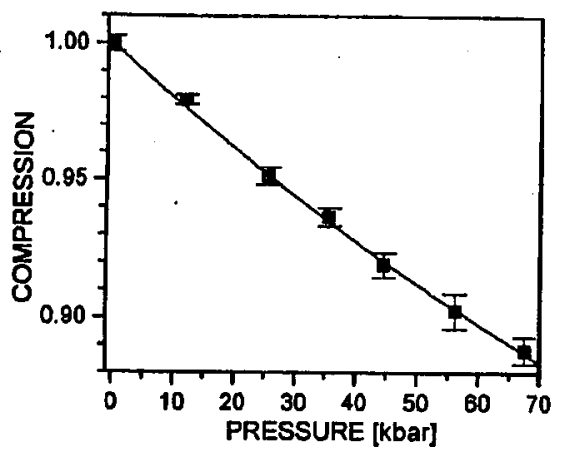

Fig. 2. Compressibility of MnTe-I as a function of pressure.

ambient [5] and intermediate [8] pressures. The obtained dependences may be approximated by

$$
\begin{aligned}
& a_{0}=3.956+1.072 \times 10^{-4} T-3.059 \times 10^{-8} T^{2}, \\
& c_{0}=6.340+3.316 \times 10^{-4} T-1.077 \times 10^{-7} T^{2}, \\
& V=85.89+9.37 \times 10^{-3} T-2.82 \times 10^{-6} T^{2}
\end{aligned}
$$

with temperature expressed in $\mathrm{K}$, lattice constants in $\AA$ and the cell volume in $\AA^{3}$.

The pressure changes of MnTe-I unit cell obtained in this work (cf. Fig. 3) are characterized by a smaller scatter of experimental points than that of earlier data $[7,8]$. The results obtained do not confirm the nonlinearity of $c_{0}$ value found in Ref. [8]. There is a small but distinct discrepancy of the slopes of the $a_{0}(p)$ and $c_{0}(p)$ among the three sets of data. The discrepancies between the present work and earlier papers on high-pressure behaviour of the lattice constants of MnTe-I are attributed to experimental errors caused by pressure and temperature drift, poor resolution and statistics, low number of measured peaks; the experimental setup
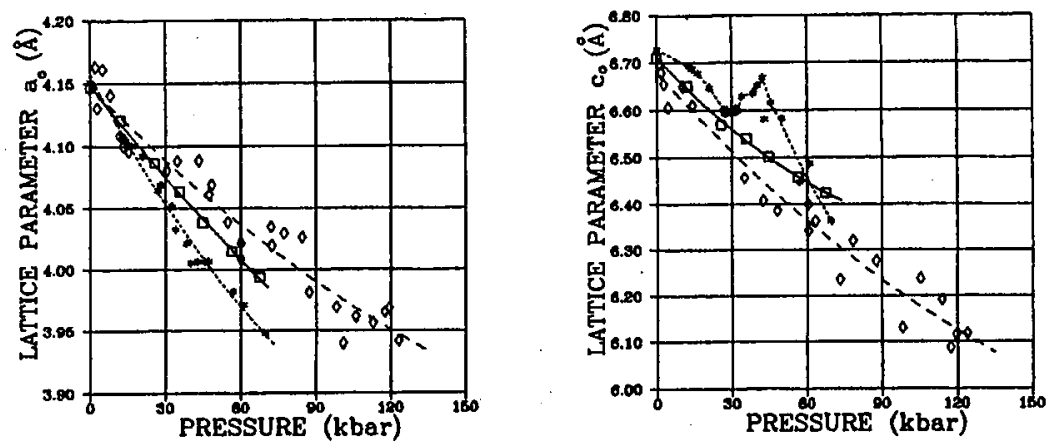

Fig. 3. Lattice parameters, $a_{0}$ and $c_{0}$, of MnTe-I as a function of pressure: this work (squares and solid line), Ref. [7] (diamonds and dashed line), Ref. [8] (stars and dotted line). 
applied in this work is thought to produce small errors compared to experiments performed in conventional laboratory conditions.

\section{Acknowledgments}

The authors are indebted to Professor Andrzej Mycielski (Institute of Physics) for the MnTe sample. Partial supports of the Committee for Scientific Research (grant No. PB 750/T08/95/09) and Deutsche Forschungsgemainschaft are gratefully acknowledged.

\section{References}

[1] J. Kossut, W. Dobrowolski, in: Handbook of Magnetic Materials, Ed. K.H.J. Buschow, Vol. 7, Elsevier, Amsterdam 1993, p. 231.

[2] N.K. Abrikosov, K.A. Dyuldina, V.V. Zhdanova, Izv. Akad. Nauk USSR - Neorg. Mater. 4, 1878 (1968).

[3] V.G. Vanyarkho, V.P. Zlomanov, A.V. Novoselova, Izv. AN SSSR - Neorg. Mater. 6, 1257 (1970).

[4] N. Kasai, Y. Nishihara, S. Ogawa, J. Phys. Soc. Jpn. 51, 452 (1982).

[5] H.P. Grazhdankina, D.P. Gurfel', Zhurn. Eksp. Teor. Fiz. 35, 907 (1958).

[6] E. Janik, E. Dynowska, J. Bąk-Misiuk, M. Leszczyński, W. Szuszkiewicz, T. Wojtowicz, G. Karczewski, A. Zakrzewski, J. Kossut, Thin Solid Films 267, 74 (1995).

[7] H. Nagasaki, I. Wakabayashi, S. Minomura, J. Phys. Chem. Solids 30, 329 (1969).

[8] H. Sugiura, A. Sawaoka, S. Saito, K. Inoue, J. Phys. Chem. Solids 40, 701 (1979).

[9] M. Mimasaka, I. Sakamoto, K. Murata, Y. Fujii, A. Onodera, J. Phys. C, Solid State Phys. 20, 4689 (1987).

[10] B. Buras, L. Gerward, in: Progress in Crystal Growth and Characterization, Ed. P. Krishna; Vol. 18, Pergamon Press, Oxford 1989, p. 93.

[11] D.L. Decker, J. Appl. Phys. 42, 3239 (1971).

[12] W. Paszkowicz, J. Appl. Crystallogr. 20, 166 (1987).

[13] W. Paszkowicz, J. Appl. Crystallogr. 22, 186 (1989).

[14] R.M. Hazen, L.W. Finger, Comparative Crystal Chemistry, John Wiley \& Sons, Chichester 1982.

[15] J.R. Buschert, F.C. Peiris, N. Samarth, H. Luo, J.K. Furdyna, Phys. Rev. B 49, 4619 (1994).

[16] P. Maheswarathan, K.J. Sladek, U. Debska, Phys. Rev. B 31, 5212 (1985). 